CLINICAL STUDY

\title{
Increased myocardial fibrosis and left ventricular dysfunction in Cushing's syndrome
}

\author{
Kai Hang Yiu ${ }^{1,2, *}$, Nina Ajmone Marsan ${ }^{1,3, *}$, Victoria Delgado ${ }^{1}$, Nienke R Biermasz ${ }^{4}$, Eduard R Holman ${ }^{1}$, \\ Johannes W A Smit ${ }^{4}$, Richard A Feelders ${ }^{5}$, Jeroen J Bax ${ }^{1}$ and Alberto M Pereira ${ }^{4}$ \\ ${ }^{1}$ Department of Cardiology, Leiden University Medical Center, Leiden, The Netherlands, ${ }^{2}$ Cardiology Division, Department of Medicine, Queen Mary \\ Hospital, University of Hong Kong, Hong Kong, China, ${ }^{3}$ The Interuniversity Cardiology Institute of the Netherlands, Utrecht, The Netherlands, \\ ${ }^{4}$ Department of Endocrinology and Metabolic Disease, Leiden University Medical Center, 2300 RC Leiden, The Netherlands and ${ }^{5}$ Section of Endocrinology, \\ Department of Internal Medicine, Erasmus Medical Center, Rotterdam, The Netherlands
}

(Correspondence should be addressed to A M Pereira; Email: a.m.pereira@lumc.nl)

*(K H Yiu and N Ajmone Marsan contributed equally to this work)

\begin{abstract}
Objective: Active Cushing's syndrome (CS) is associated with cardiomyopathy, characterized by myocardial structural, and ultrastructural abnormalities. The extent of myocardial fibrosis in patients with CS has not been previously evaluated. Therefore, the objective of this study was to assess myocardial fibrosis in CS patients, its relationship with left ventricular (LV) hypertrophy and function, and its reversibility after surgical treatment.

Design and methods: Fifteen consecutive CS patients $(41 \pm 12$ years) were studied together with 30 hypertensive (HT) patients (matched for LV hypertrophy) and 30 healthy subjects. Echocardiography was performed in all patients including i) LV systolic function assessment by conventional measures and by speckle tracking-derived global longitudinal strain, ii) LV diastolic function assessment using E/E', and iii) myocardial fibrosis assessment using calibrated integrated backscatter (IBS). Echocardiography was repeated after normalization of cortisol secretion (14 3 months).

Results: CS patients showed the highest value of calibrated IBS $(-15.1 \pm 2.5 \mathrm{~dB})$ compared with HT patients $(-20.0 \pm 2.6 \mathrm{~dB}, P<0.01)$ and controls $(-23.8 \pm 2.4 \mathrm{~dB}, P<0.01)$, indicating increased myocardial fibrosis independent of LV hypertrophy. Moreover, calibrated IBS in CS patients was significantly related to both diastolic function $\left(\mathrm{E} / \mathrm{E}^{\prime}, r=0.79, P<0.01\right)$ and systolic function (global longitudinal strain, $r=0.60, P=0.02$ ). After successful surgical treatment, calibrated IBS normalized $(-21.0 \pm 3.8$ vs $-15.1 \pm 2.5 \mathrm{~dB}, P<0.01)$, suggestive of regression of myocardial fibrosis.

Conclusions: Patients with CS have increased myocardial fibrosis, which is related to LV systolic and diastolic dysfunction. Successful treatment of CS normalizes the extent of myocardial fibrosis. Therefore, myocardial fibrosis appears to be an important factor in the development and potential regression of CS cardiomyopathy.
\end{abstract}

European Journal of Endocrinology 166 27-34

\section{Introduction}

Patients with Cushing's syndrome (CS) have an increased risk for cardiovascular diseases (1). Previous studies using echocardiography have demonstrated that CS is associated with a specific cardiomyopathy, characterized by left ventricular (LV) hypertrophy, increased relative wall thickness (2), and both diastolic (3) and systolic dysfunction $(4,5)$. These alterations are related to the endogenous glucocorticoid excess, which leads to an increase in blood pressure via the stimulation of both mineralocorticoid (MR) and glucocorticoid receptors (GR) (6-8) but also through the activation of the renin-angiotensin system components and through the effect of cortisol on peripheral and systemic vasculature (9-11). These mechanisms are also proposed to be crucial in the development of myocardial fibrosis and significant structural damage $(12,13)$. However, myocardial tissue alterations, and in particular myocardial fibrosis in CS patients, have not been previously evaluated.

Ultrasonic integrated backscatter (IBS) derived by two-dimensional (2D) echocardiography provides information on myocardial ultrasound reflectivity, which increases when myocardial fibrosis increases $(14,15)$. Fibrosis assessment using IBS has been validated in a direct comparison with histological findings (14) and it has been used in detecting increased myocardial fibrosis in several cardiovascular diseases, including hypertensive (HT) patients $(16,17)$. 
Therefore, we hypothesized that i) myocardial fibrosis in CS, as measured by IBS, would be increased and ii) that the extent of myocardial fibrosis would be significantly related to $L V$ function. Finally, we hypothesized that, if this would be the case, successful treatment of CS would lead to regression or even normalization of the extent of myocardial fibrosis.

\section{Subjects and methods}

\section{Patient population}

The patient population consisted of 15 consecutive CS patients, who were referred to the Endocrinology Department of our Center and whose clinical and echocardiographic characteristics have been previously described (5). Briefly, the underlying cause of CS was an ACTH-producing pituitary adenoma in 12 patients, while in three patients CS was ACTH independent. The diagnosis of CS was made on clinical grounds in combination with the following biochemical criteria: increased $24 \mathrm{~h}$ urinary free cortisol excretion $(>220 \mathrm{nmol})$, failure of serum cortisol to suppress after low-dose dexamethasone (one evening dose of $1 \mathrm{mg}$ ), and loss of diurnal rhythm. In case of ACTH-dependent CS, the suppression of serum cortisol during a $7 \mathrm{~h}$ i.v. dexamethasone suppression test $(5,18)$ was also evaluated together with the response of serum cortisol and ACTH on i.v. corticotropin-releasing hormone stimulation (19).

All patients underwent surgical treatment (transsphenoidal adenomectomy or unilateral or bilateral adrenalectomy) followed by pituitary irradiation when necessary $(n=6)$. After treatment, all patients were assessed at least twice yearly by an endocrinologist, with adequate evaluation and treatment of possible pituitary hormone deficiencies. Patients were considered in remission according to normal $24 \mathrm{~h}$ urinary cortisol excretion rates $(<80 \mu \mathrm{g} / 24 \mathrm{~h}$ or $<220 \mathrm{nmol} / 24 \mathrm{~h})$ and normal overnight suppression of serum cortisol $(<1.8 \mu \mathrm{g} / \mathrm{dl}$ or $<50 \mathrm{nmol} / \mathrm{l})$ after $1 \mathrm{mg}$ dexamethasone.

\section{Study protocol}

All patients underwent transthoracic echocardiography at baseline and at least 1 year and maximum 18 months (median $14 \pm 2.8$ months) after obtaining definite biochemical remission either by surgery alone or by surgery followed by radiotherapy. The echocardiographic examination included

i) conventional measurements of $\mathrm{LV}$ size and $\mathrm{LV}$ systolic and diastolic function;

ii) 2D speckle tracking strain analysis, which is a highly sensitive echocardiographic technique for the assessment of subtle changes of LV systolic function (5); and

iii) calibrated IBS, for the assessment of myocardial fibrosis.
By using these echocardiographic techniques, the relationship between the changes in LV function and in myocardial fibrosis after surgical treatment could be addressed.

For comparison, 30 healthy individuals (2:1 ratio to CS patients) matched for age and gender were consecutively selected as a control group from an existing echocardiographic database with this information. These subjects were referred for atypical chest pain, palpitations, or syncope without murmur and showed normal structural heart on echocardiography. Furthermore, they were carefully screened to exclude the presence of HT (systolic blood pressure $<130 \mathrm{mmHg}$ and/or diastolic blood pressure $<90 \mathrm{mmHg}$ ) or any history of anti-HT treatment. In addition, in order to evaluate whether an increased myocardial fibrosis in CS patients is intrinsic to the disease or mainly related to the presence of $\mathrm{LV}$ hypertrophy, 30 patients with essential HT (2:1 ratio to patients with CS) matched for end-diastolic septal wall thickness and LV mass index were also recruited for comparison from the same echocardiographic database.

The study was approved by the local institutional ethics committee, and written informed consent was obtained from all patients and subjects.

\section{Echocardiography}

All patients were imaged in the left lateral decubitus position using a commercially available system (Vingmed Vivid 7, General Electric Vingmed Ultrasound, Horten, Norway). Images were obtained using a $3.5 \mathrm{MHz}$ transducer and digitally stored in cine-loop format; offline analysis was performed using EchoPAC version 108.1.5 (GE-Vingmed Ultrasound). From the standard apical views (4- and 2-chamber), LV volumes and ejection fraction were calculated using Simpson's biplane method (14). LV end-diastolic and end-systolic diameter and LV septum and posterior wall thickness were measured from M-mode recordings obtained at the parasternal long-axis view. In addition, relative wall thickness was calculated as the ratio between enddiastolic posterior wall thickness and LV diameter (20), and LV mass index was calculated by Devereux's formula and indexed to body surface area (21).

Diastolic function was evaluated by measuring transmitral flow E-wave velocity (E) and A-wave velocity (A) using pulse-wave Doppler recordings, and the E/A ratio was calculated (22). In addition, as an estimate of LV filling pressure, early diastolic peak velocity $\left(\mathrm{E}^{\prime}\right)$ was measured at the septal level of mitral valve annulus from tissue Doppler imaging data, and $\mathrm{E} / \mathrm{E}^{\prime}$ ratio was derived (23).

\section{D speckle tracking strain analysis}

The measure of global longitudinal, radial, and circumferential strains using speckle tracking has 

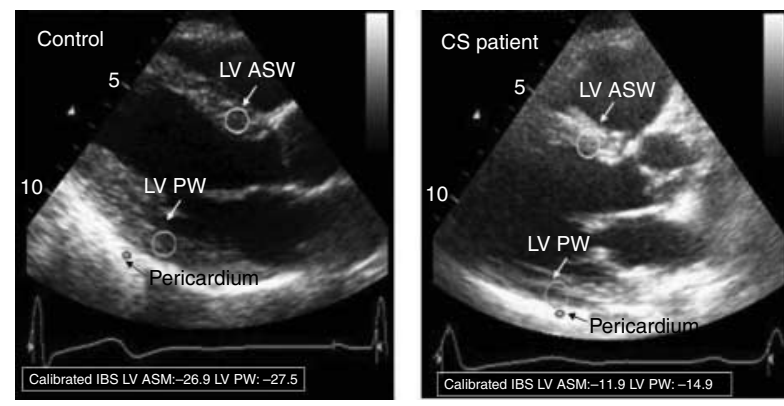

Figure 1 Examples of left ventricular (LV) myocardial fibrosis assessment using calibrated integrated backscatter (IBS). At enddiastole, a fixed region of interest is positioned at the level of LV anteroseptal wall (LV ASW), posterior wall (LV PW), and in the pericardium. In the left panel, an example of a normal subject with mean calibrated IBS $-27.2 \mathrm{~dB}$. The right panel shows an example of a patient with Cushing's syndrome (CS) with a mean IBS of $-13.4 \mathrm{~dB}$, demonstrating increased myocardial fibrosis compared with the normal subject.

been previously described (5). Briefly, global longitudinal strain is obtained from the apical 2-chamber, 4-chamber, and long-axis views as the average of 18 segmental (six from each view) peak strain values, and it evaluates the shortening/lengthening of myocardial wall. From the LV short-axis view, thickening/thinning of the myocardial wall can be assessed with radial strain, whereas the myocardial deformation along the curvature of the LV can be evaluated with circumferential strain. The mid-ventricular short-axis of the $L V$ is divided into six segments and the global values of radial and circumferential strains are derived from the average of the segmental peak strain values. The intra- and inter-observer agreements for the measurement of radial, circumferential, and longitudinal strain have been reported previously (5).

\section{Fibrosis assessment with calibrated IBS}

Calibrated IBS evaluates myocardial ultrasound reflectivity based on gray-scale 2D images. Within the heart, the pericardium contains the highest content of fibrosis and shows the highest ultrasound reflectivity, whereas blood pool is characterized by the lowest ultrasound reflectivity. The myocardium has an intermediate ultrasound reflectivity, which reflects the degree of myocardial fibrosis $(14,15,24)$. The IBS measurements were obtained from the parasternal long-axis view with a frame rate between 80 and 120 frames/s. Three cardiac cycles were stored in cine-loop format for offline analysis (EchoPAC version 108.1.5). A $9 \times 9 \mathrm{~mm}$ region of interest was positioned in the mid-myocardium of the anteroseptal and posterior wall of the $\mathrm{LV}$ and a $2 \times 3$ $\mathrm{mm}$ region of interest was positioned in the pericardium for reference. The measurements of IBS intensity were performed at end-diastole and expressed in $\mathrm{dB}$. Calibrated IBS was calculated by subtracting pericardial IBS intensity from myocardial IBS intensity of the LV anteroseptal and posterior wall and the mean value of the two walls was used. Examples of calibrated IBS measurement in a normal subject and a CS patient are shown in Fig. 1. The intra- and inter-observer agreements for calibrated IBS (calculated by re-measuring the entire patient population) were 0.91 and 0.92 , respectively, using intra-class correlation coefficients.

\section{Statistical analysis}

Continuous variables are presented as mean \pm s.D. Categorical data are presented as frequencies and percentages. One-way ANOVA with post hoc test by Bonferroni was used to examine differences among groups. Pearson's correlation test was used to evaluate the correlation between calibrated IBS and other echocardiographic parameters. Mean calibrated IBS was compared before and after surgical treatment of CS using paired $t$-test. All statistical analyses were performed using the statistical package SPSS for windows (Version 15.0, SPSS, Chicago, IL, USA). A P value $<0.05$ was considered to be statistically significant.

\section{Results}

\section{Baseline clinical characteristics}

Baseline clinical characteristics of CS patients, HT patients, and healthy controls are shown in Table 1. According to the matching criteria, age was similar between controls and CS patients, who were both significantly younger than the HT patients. All three groups had similar gender distribution. Body mass index was significantly higher in HT and CS patients

Table 1 Baseline clinical characteristics of healthy controls, HT and CS patients.

\begin{tabular}{lcccr}
\hline & $\begin{array}{c}\text { Controls } \\
(n=30)\end{array}$ & $\begin{array}{c}\text { HT } \\
\text { patients } \\
(n=30)\end{array}$ & $\begin{array}{c}\text { CS } \\
\text { patients } \\
(n=15)\end{array}$ & $\begin{array}{r}\text { ANOVA } \\
\text { P value }\end{array}$ \\
\hline Age (years) & $40 \pm 8$ & $53 \pm 13^{*}$ & $41 \pm 12^{\dagger}$ & $<\mathbf{0 . 0 1}$ \\
Gender - male (\%) & $14(46.7)$ & $15(50.0)$ & $6(40.0)$ & 0.96 \\
BMI (kg/m²) & $23.8 \pm 3.1$ & $27.0 \pm 5.4^{*}$ & $27.4 \pm 4.7^{*}$ & $<\mathbf{0 . 0 1}$ \\
SBP (mmHg) & $120 \pm 9$ & $138 \pm 12^{*}$ & $135 \pm 13^{*}$ & $<\mathbf{0 . 0 1}$ \\
DBP (mmHg) & $75 \pm 10$ & $86 \pm 9^{*}$ & $88 \pm 12^{*}$ & $<\mathbf{0 . 0 1}$ \\
ACEI (\%) & - & $6(20.0)$ & $1(6.7)$ & 0.40 \\
ARB (\%) & - & $4(13.3)$ & $1(6.7)$ & 0.65 \\
Calcium channel & - & $5(16.7)$ & $1(6.7)$ & 0.65 \\
$\quad$ blockers (\%) & & & & \\
Diuretics (\%) & - & $6(20.0)$ & $1(6.7)$ & 0.40 \\
$\beta$-Blockers (\%) & - & $10(33.3)$ & $3(20)$ & 0.49 \\
Anti-diabetics/ & - & - & $5(33 \%)$ & - \\
$\quad$ insulin (\%) & & & & \\
\hline
\end{tabular}

BMI, body mass index; ACEI, angiotensin converting enzyme inhibitor; ARB, angiotensin receptor blocker; DBP, diastolic blood pressure; $\mathrm{SBP}$, systolic blood pressure. ANOVA $P$ values $<0.05$ are in bold. *Significant differences with controls; ${ }^{\dagger}$ Significant differences with HT patients. 
Table 2 Baseline echocardiographic characteristics of controls, HT and CS patients.

\begin{tabular}{lcccr}
\hline & $\begin{array}{c}\text { Controls } \\
(n=30)\end{array}$ & $\begin{array}{c}\text { HT patients } \\
(n=30)\end{array}$ & $\begin{array}{c}\text { CS patients } \\
(n=15)\end{array}$ & $\begin{array}{r}\text { ANOVA } \\
\boldsymbol{P} \text { value }\end{array}$ \\
\hline LV end-diastolic diameter (cm) & $4.7 \pm 0.3$ & $4.5 \pm 0.3$ & $5.0 \pm 0.6$ & 0.27 \\
LV end-systolic diameter (cm) & $3.1 \pm 0.3$ & $2.9 \pm 0.2$ & $3.2 \pm 0.5$ & 0.31 \\
LV ejection fraction (\%) & $60 \pm 4$ & $62 \pm 5$ & $59 \pm 9$ & 0.21 \\
End-diastolic septal thickness (cm) & $0.9 \pm 0.1$ & $1.2 \pm 0.3^{*}$ & $1.3 \pm 0.2^{*}$ & $<\mathbf{0 . 0 1}$ \\
LV mass index (g/m $\left.{ }^{2}\right)$ & $79.6 \pm 16.1$ & $124.1 \pm 35.2^{*}$ & $125.6 \pm 37.2^{*}$ & $<\mathbf{0 . 0 1}$ \\
Relative wall thickness & $0.37 \pm 0.06$ & $0.49 \pm 0.09^{*}$ & $0.48 \pm 0.09^{*}$ & $<\mathbf{0 . 0 1}$ \\
E wave velocity (m/s) & $78.8 \pm 12.6$ & $73.1 \pm 14.1$ & $58.2 \pm 12.6^{*, \dagger}$ & $<\mathbf{0 . 0 1}$ \\
A wave velocity (m/s) & $58.6 \pm 14.2$ & $73.2 \pm 16.8^{*}$ & $65.0 \pm 22.0$ & $<\mathbf{0 . 0 1}$ \\
E/A ratio & $1.4 \pm 0.5$ & $1.1 \pm 0.3^{*}$ & $1.0 \pm 0.4^{*}$ & $<\mathbf{0 . 0 1}$ \\
E $^{\prime}$ velocity (cm/s) & $10.0 \pm 2.4$ & $8.3 \pm 3.4^{*}$ & $6.0 \pm 2.4^{*}$ & $<\mathbf{0 . 0 1}$ \\
E/E' ratio & $8.0 \pm 1.9$ & $10.3 \pm 4.3^{*}$ & $10.9 \pm 3.8^{*}$ & $\mathbf{0 . 0 1}$ \\
Global longitudinal strain (\%) & $-21.4 \pm 1.9$ & $-19.3 \pm 2.2^{*}$ & $-15.4 \pm 1.9^{*}+$ & $<\mathbf{0 . 0 1}$ \\
Global circumferential strain (\%) & $-20.9 \pm 2.5$ & $-20.7 \pm 2.1$ & $-16.5 \pm 3.5^{*}$, & $<\mathbf{0 . 0 1}$ \\
Global radial strain (\%) & $38.8 \pm 13.7$ & $38.6 \pm 14.5$ & $33.8 \pm 12.9$ & 0.53 \\
\hline
\end{tabular}

A, trans-mitral A-wave velocity; E, trans-mitral E-wave velocity; $E^{\prime}$, early diastolic peak velocity; LV, left ventricular. *Significant differences with controls; ${ }^{\dagger}$ Significant differences with HT patients. ANOVA $P$ values $<0.05$ are in bold.

compared with healthy controls. As expected, CS and HT patients had higher values of systolic and diastolic blood pressure compared with healthy subjects. Of note, $45 \%$ of CS patients received anti-HT medication, including angiotensin converting enzyme inhibitors, angiotensin receptor blockers, calcium channel blockers, diuretics, and $\beta$-blockers.

\section{Baseline echocardiography and $2 D$ speckle tracking strain analysis}

According to the matching criteria, HT and CS patients showed comparable end-diastolic septal thickness and LV mass index, which were larger compared with normal subjects (Table 2). No significant differences in $\mathrm{LV}$ dimensions and $\mathrm{LV}$ ejection fraction were observed between the three groups. Diastolic function, as measured by $\mathrm{E} / \mathrm{A}$ ratio and $\mathrm{E} / \mathrm{E}^{\prime}$ ratio, was significantly impaired in CS and HT patients compared with the control group.

Speckle tracking strain analysis for the assessment of cardiac systolic function showed significant impairment of global longitudinal strain in the HT and CS groups compared with controls (Table 2). In particular, global longitudinal strain was significantly lower in the CS group compared with the HT group. Global circumferential strain was significantly lower in CS patients, while no differences were noted between controls and HT patients. Global radial strain showed no significant differences among the three groups.

\section{Myocardial fibrosis assessment with calibrated IBS}

Patients with CS showed the highest mean calibrated IBS $(-15.1 \pm 2.5 \mathrm{~dB})$ compared with HT patients $(-20.0 \pm 2.6 \mathrm{~dB}, \quad P<0.01)$ and controls $(-23.8$ $\pm 2.4 \mathrm{~dB}, P<0.01$; Fig. 2), suggesting an increased myocardial fibrosis. Moreover, mean calibrated IBS was significantly higher in HT patients compared with healthy subjects $(P<0.01)$.

Mean calibrated IBS in CS patients was significantly related to $\mathrm{LV}$ diastolic function, namely with $\mathrm{E} / \mathrm{E}^{\prime}$ ratio $(r=0.79, P<0.01)$, and LV systolic function, i.e. global longitudinal strain $(r=0.60, P=0.02$; Table 3$)$. In turn, no significant relation was observed with global circumferential or radial strain, age, blood pressure, or LV mass index.

\section{Follow-up of the patient with CS after surgery}

All patients were in biochemical remission for at least 1 year at follow-up, but six patients remained glucocorticoid dependent after surgery (hydrocortisone dose was on average $20 \mathrm{mg}$ /day divided into three dosages). The number of patients using anti-HT or anti-diabetic

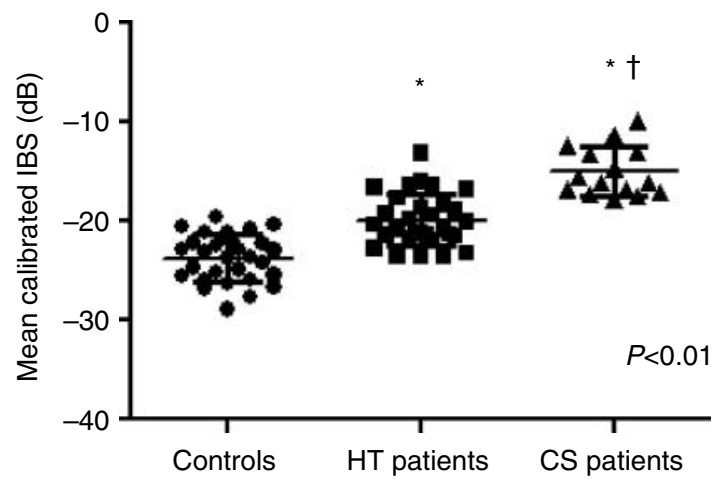

Figure 2 Comparison of mean calibrated integrated backscatter (IBS) between normal subjects (controls), hypertensive (HT) patients, and patients with Cushing's syndrome (CS) before treatment. Patients with CS show the highest mean calibrated IBS compared with HT patients and controls, suggestive of increased myocardial fibrosis. *Significant differences with controls;

${ }^{\dagger}$ Significant differences with HT patients. 
Table 3 Correlation coefficient $(r)$ between mean calibrated integrated backscatter (IBS) and clinical and echocardiographic parameters in patients with CS.

\begin{tabular}{lrr}
\hline & \multicolumn{2}{c}{ CS patients } \\
\cline { 2 - 3 } & \multicolumn{1}{c}{$r$} & $P$ value \\
\hline Age & 0.27 & 0.33 \\
Body mass index & -0.40 & 0.13 \\
Urine free cortisol & 0.32 & 0.36 \\
SBP & -0.42 & 0.12 \\
DBP & -0.27 & 0.33 \\
LV mass index & -0.07 & 0.81 \\
LV ejection fraction & -0.28 & 0.34 \\
LV end-diastolic diameter & -0.19 & 0.72 \\
E wave velocity & 0.16 & 0.55 \\
E/A ratio & -0.29 & 0.29 \\
E' velocity & -0.63 & $\mathbf{0 . 0 2}$ \\
E/E' ratio & 0.79 & $\mathbf{0 . 0 1}$ \\
Global longitudinal strain & 0.60 & $\mathbf{0 . 0 2}$ \\
Global circumferential strain & 0.37 & 0.19 \\
Global radial strain & -0.39 & 0.17 \\
\hline
\end{tabular}

For abbreviations, see Tables 1 and 2. $P$ values $<0.05$ are in bold.

medication at follow-up reduced from six to four and from five to three patients respectively. Before treatment, all six premenopausal female patients had secondary amenorrhea and another two patients (both with pituitary macroadenoma) had additional hormone pituitary insufficiency (both GH deficiency and secondary hypothyroidism). After surgery, a regular menstrual cycle after obtaining remission was restored in all six women. GH deficiency and secondary hypogonadism persisted in two patients after treatment. Other treatment-induced deficiencies, besides hydrocortisone dependency, were not documented during the follow-up period.

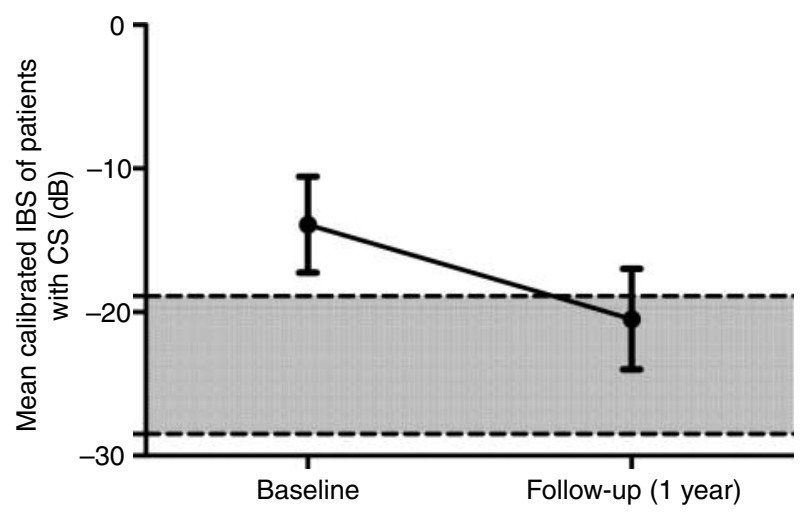

Figure 3 Mean calibrated integrated backscatter (IBS) in patients with Cushing's syndrome (CS) before (baseline) and after surgical treatment (follow-up). The shaded area represents the normal range of mean calibrated IBS, derived from the control group as the mean value \pm 2 s.D. Upon successful surgical treatment, the mean calibrated IBS in CS patients significantly decreased $(P<0.01)$ and reached the range of normal values, suggesting the reversibility of myocardial fibrosis.
As described previously (5), after surgical treatment and complete biochemical remission, a significant regression of LV hypertrophy with a reduction in enddiastolic septum thickness $(1.3 \pm 0.2$ vs $1.0 \pm 0.2 \mathrm{~cm}$, $P<0.01)$, LV mass index $\left(126 \pm 37\right.$ vs $98 \pm 31 \mathrm{~g} / \mathrm{m}^{2}$, $P=0.01)$, and relative wall thickness $(0.48 \pm 0.09$ vs $0.41 \pm 0.08 \mathrm{~cm}, P=0.04)$ was observed in patients with CS. Furthermore, significant improvements were observed in $\mathrm{E}^{\prime}$ velocity $(6.0 \pm 2.4$ vs $8.2 \pm 2.6 \mathrm{~cm} / \mathrm{s}$, $P=0.04)$, global longitudinal strain $(-15.4 \pm 1.9$ vs $-18.9 \pm 2.1 \%, P<0.01)$, and global circumferential strain $(-16.5 \pm 3.5$ vs $-20.3 \pm 3.9 \%, P<0.01)$, but not in global radial strain $(33.8 \pm 12.8$ vs 30.6 $\pm 11.6 \%, P=0.75$ ).

A significant reduction of IBS to values comparable to healthy controls was observed at follow-up $(-15.1$ \pm 2.5 vs $-21.0 \pm 3.8 \mathrm{~dB}, P<0.01)$, suggestive for a decrease in myocardial fibrosis after successful surgical treatment of CS. (Fig. 3).

\section{Discussion}

The results of the current study demonstrate that myocardial fibrosis is significantly increased in active, untreated patients with CS compared with both healthy subjects and HT patients, pointing toward a direct effect of cortisol excess on myocardial fibrosis, independent of LV hypertrophy and blood pressure. Furthermore, in patients with CS, mean calibrated IBS significantly correlated with LV systolic (global longitudinal strain) and diastolic function $\left(\mathrm{E} / \mathrm{E}^{\prime}\right)$, indicating that myocardial fibrosis per se affects cardiac function. Finally, the extent of myocardial fibrosis normalized upon successful treatment of CS. Therefore, myocardial fibrosis appears to be an important factor both in the development and in the regression of the cardiomyopathy as seen in CS.

Cardiac involvement in patients with CS is mainly characterized by the presence of LV hypertrophy and concentric remodeling $(2,4)$. These structural abnormalities can be partially explained by the rise in blood pressure, but the most important role is attributed to glucocorticoid excess, which directly activates some of the components of the rennin-angiotensin system (25, 26 ) and in particular enhances angiotensin-II responsiveness of the myocytes (27). More importantly, control of both the MRs and the GRs appears to be critical in the regulation and development of myocardial fibrosis (28, 29). Under physiological circumstances, circulating cortisol levels are more than 100-fold higher than aldosterone. Because MRs have equal affinity for aldosterone and cortisol, selective activation of MRs by aldosterone in epithelial tissue and vascular smooth muscle cells is achieved by co-expression of the enzyme 11 $\beta$-hydroxysteroid dehydrogenase type 2 (11ßHSD2) that converts endogenous cortisol to its MR-inactive cortisone. Under normal circumstances, $11 \beta \mathrm{HSD} 2$ is not expressed in cardiomyocytes (30) and therefore MRs 
are overwhelmingly occupied by normal cortisol levels, acting as an MR antagonist. However, in the presence of tissue damage, cortisol can act as an MR agonist (31), and MR and 11ßHSD2 co-expression has recently been demonstrated in human perfused hearts (12). It is unknown, however, whether these experimental findings can be extrapolated to the clinical situation as seen in endogenous CS.

Collagen deposition increases myocardial acoustic impedance and reflectivity, which can be detected by calibrated IBS (14). This technique has been previously used to evaluate myocardial fibrosis in several cardiovascular diseases $(16,32)$. In a study of 117 HT patients referred for an echocardiographic evaluation, Di Bello et al. (17) showed that HT patients had higher IBS values than normal controls and that IBS was significantly correlated with the severity of LV hypertrophy. This study demonstrated that patients with active CS have increased myocardial fibrosis as assessed by calibrated IBS, when compared with both normal subjects and HT patients with similar LV wall thickness and mass index. These findings are in agreement with a direct deleterious effect of cortisol excess on the myocardium, superimposed and independent of elevated blood pressure, and LV hypertrophy.

Previous studies have shown the relationship between the extent of myocardial fibrosis and the degree of myocardial dysfunction in various cardiac diseases $(16,32)$. Shan et al. (33) demonstrated that in patients with coronary artery disease, reduced myocardial velocities and impaired LV relaxation assessed by echocardiography were inversely related to the percentage of interstitial fibrosis quantified on endomyocardial biopsies. Similarly, the results of the current study demonstrated that in patients with CS, myocardial fibrosis, as measured by IBS, was significantly related to both LV systolic function, assessed by global longitudinal strain, and to LV diastolic function. However, neither blood pressure nor LV mass index was significantly related to calibrated IBS. These observations point toward specific cortisol excess-induced effects on the development of myocardial fibrosis that, in turn, significantly contributes to the development of cardiac dysfunction, independent of LV hypertrophy. In particular, global longitudinal strain, reflecting myocardial shortening of the subendocardial layer, is highly sensitive to subtle changes in LV systolic function potentially mediated by increased myocardial fibrosis. Conversely, impairment of circumferential and radial strain showed to occur in a more advanced stage of cardiac involvement and to be less sensitive for the detection of subtle changes in LV function. Furthermore, myocardial fibrosis is also one of the main determinants of $\mathrm{LV}$ wall stiffness and therefore directly affects $\mathrm{LV}$ diastolic function, as measured by $\mathrm{E} / \mathrm{E}^{\prime}$. However, the exact mechanism underlying the relationship between structural and functional myocardial alterations requires further evaluations.
A significant reduction in $\mathrm{LV}$ mass, relative wall thickness, and improvement in both diastolic and systolic function has been shown after successful surgical treatment for CS (5). This study has also demonstrated that normalization of cortisol levels in patients with CS leads to a significant reduction of myocardial fibrosis detected by calibrated IBS. As previously shown in HT patients treated with renninangiotensin system antagonists $(34,35)$, reduction in angiotensin-II activity may i) significantly reduce myocardial collagen synthesis, as demonstrated by a drop in serum concentrations of procollagens (36) and ii) enhance collagen degradation (37), resulting in a significant regression of myocardial fibrosis. Accordingly, normalization of cortisol levels after successful treatment of CS should not only normalize the activity of some of the renin-angiotensin system components but also the regulation of tissue-specific MR and GR, resulting in reversal of increased myocardial fibrosis.

The assessment of the extent of myocardial fibrosis by calibrated IBS represents a novel approach to detect cardiac involvement in patients with CS, eventually before the occurrence of subtle myocardial dysfunction. Therefore, it might be used for patient management and monitoring. In addition, this study, prospectively evaluating patients with CS before and after successful treatment, represents a clinical model that confirms the significant effect of glucocorticoid excess on myocardial fibrosis.

In conclusion, patients with active CS have increased myocardial fibrosis, which is related to LV systolic and diastolic dysfunction. Successful treatment of CS normalizes the extent of myocardial fibrosis parallel to LV systolic and diastolic function. Whether these observations also apply to patients treated with exogenous corticosteroids for other, more prevalent diseases, like chronic obstructive pulmonary disease, or rheumatoid arthritis, still remains to be determined.

\section{Declaration of interest}

J J Bax receives grants from Biotronik, Lantheus medical imaging, Boston Scientific, Edwards Lifesciences, GE Healthcare, Medtronic and St Jude Medical. A M Pereira received consultancy fees from Pfizer, and $R$ A Feelders from Novartis. The remaining authors have no conflict of interest.

\section{Funding}

K H Yiu is financially supported by a research grant from the Hong Kong Heart Foundation.

\section{Acknowledgements}

We thank Drs Arnold C T Ng, Tomasz G Witkowski, See Hooi Ewe, and Dominique Auger at the Department of Cardiology, Leiden University Medical Center, for the help provided in data collection and Prof. Hung Fat Tse at the Department of Medicine, University of Hong Kong, for reviewing the manuscript. 


\section{References}

1 Colao A, Pivonello R, Spiezia S, Faggiano A, Ferone D, Filippella M, Marzullo P, Cerbone G, Siciliani M \& Lombardi G. Persistence of increased cardiovascular risk in patients with Cushing's disease after five years of successful cure. Journal of Clinical Endocrinology and Metabolism $1999 \mathbf{8 4}$ 2664-2672. (doi:10.1210/jc.84.8. 2664)

2 Fallo F, Budano S, Sonino N, Muiesan ML, Agabiti-Rosei E \& Boscaro M. Left ventricular structural characteristics in Cushing's syndrome. Journal of Human Hypertension 19948 509-513.

3 Baykan M, Erem C, Gedikli O, Hacihasanoglu A, Erdogan T, Kocak M, Kaplan S, Kiriş A, Orem C \& Celik S. Assessment of left ventricular diastolic function and Tei index by tissue Doppler imaging in patients with Cushing's syndrome. Echocardiography 200825 182-190. (doi:10.1111/j.1540-8175.2007.00572.x)

4 Muiesan ML, Lupia M, Salvetti M, Grigoletto C, Sonino N, Boscaro M, Rosei EA, Mantero F \& Fallo F. Left ventricular structural and functional characteristics in Cushing's syndrome. Journal of the American College of Cardiology $2003412275-2279$. (doi:10.1016/S0735-1097(03)00493-5)

5 Pereira AM, Delgado V, Romijn JA, Smit JW, Bax JJ \& Feelders RA. Cardiac dysfunction is reversed upon successful treatment of Cushing's syndrome. European Journal of Endocrinology 2010162 331-340. (doi:10.1530/EJE-09-0621)

6 Fraser R, Davies DL \& Connell JMC. Hormones and hypertension. Clinical Endocrinology 198931 701-746. (doi:10.1111/j.13652265.1989.tb01295.x)

7 Whitworth JA, Gordon D, Andrews J \& Scoggins BA. The hypertensive effect of synthetic glucocorticoids in man: role of sodium and volume. Journal of Hypertension 19897 537-549. (doi:10.1097/00004872-198907000-00005)

8 Nieman LK, Chrousos GP, Kellner C, Spitz IM, Nisula BC, Cutler GB, Merriam GR, Bardin CW \& Loriaux DL. Successful treatment of Cushing's syndrome with the glucocorticoid antagonist RU 486 . Journal of Clinical Endocrinology and Metabolism 198561 536-540. (doi:10.1210/jcem-61-3-536)

9 Suzuki H, Handa M, Kondo K \& Saruta T. Role of reninangiotensin system in glucocorticoid hypertension in rats. American Journal of Physiology. Endocrinology and Metabolism 1982243 E48-E51.

10 Magiakou MA, Smyrnaki P \& Chrousos GP. Hypertension in Cushing's syndrome. Best Practice \& Research. Clinical Endocrinology \& Metabolism 200620 467-482. (doi:10.1016/j. beem.2006.07.006)

11 Sharma ST \& Nieman LK. Cushing's syndrome: all variants, detection, and treatment. Endocrinology and Metabolism Clinics of North America 201140 379-391. (doi:10.1016/j.ecl.2011.01. 006)

12 Chai W, Hofland J, Jansen PM, Garrelds IM, de Vries R, van den Bogaerdt AJ, Feelders RA, de Jong FH \& Danser AH. Steroidogenesis vs. steroid uptake in the heart: do corticosteroids mediate effects via cardiac mineralocorticoid receptors? Journal of Hypertension 201028 1044-1053. (doi:10.1097/HJH.0b013e $328335 \mathrm{c} 381$ )

13 Scheuer DA \& Bechtold AG. Glucocorticoids potentiate central actions of angiotensin to increase arterial blood pressure. American Journal of Physiology. Regulatory, Integrative and Comparative Physiology 2001280 R1719-R1726.

14 Picano E, Pelosi G, Marzilli M, Lattanzi F, Benassi A, Landini L \& L'Abbate A. In vivo quantitative ultrasonic evaluation of myocardial fibrosis in humans. Circulation 199081 58-64. (doi:10.1161/01.CIR.81.1.58)

15 Pérez JE, Barzilai B, Madaras EI, Glueck RM, Saffitz JE, Johnston P, Miller JG \& Sobel BE. Applicability of ultrasonic tissue characterization for longitudinal assessment and differentiation of calcification and fibrosis in cardiomyopathy. Journal of the American College of Cardiology 19844 88-95. (doi:10.1016/S0735-1097(84) 80323-X)
16 Fang ZY, Yuda S, Anderson V, Short L, Case C \& Marwick TH. Echocardiographic detection of early diabetic myocardial disease. Journal of the American College of Cardiology 200341 611-617. (doi:10.1016/S0735-1097(02)02869-3)

17 Di Bello V, Giorgi D, Talini E, Dell' Omo G, Palagi C, Romano MF, Pedrinelli R \& Mariani M. Incremental value of ultrasonic tissue characterization (backscatter) in the evaluation of left ventricular myocardial structure and mechanics in essential arterial hypertension. Circulation $2003 \mathbf{1 0 7}$ 74-80. (doi:10.1161/01. CIR.0000041045.26774.1C)

18 Biemond P, de Jong FH \& Lamberts SW. Continuous dexamethasone infusion for seven hours in patients with the Cushing syndrome. A superior differential diagnostic test. Annals of Internal Medicine $1990112738-742$.

19 Newell-Price J, Trainer P, Besser M \& Grossman A. The diagnosis and differential diagnosis of Cushing's syndrome and pseudoCushing's states. Endocrine Reviews 199819 647-672. (doi:10. 1210/er.19.5.647)

20 Lang RM, Bierig M, Devereux RB, Flachskampf FA, Foster E, Pellikka PA, Picard MH, Roman MJ, Seward J, Shanewise JS, Solomon SD, Spencer KT, Sutton MS \& Stewart WJ; Chamber Quantification Writing Group; American Society of Echocardiography's Guidelines and Standards Committee; European Association of Echocardiography. Recommendations for chamber quantification: a report from the American Society of Echocardiography's Guidelines and Standards Committee and the Chamber Quantification Writing Group, developed in conjunction with the European Association of Echocardiography, a branch of the European Society of Cardiology. Journal of the American Society of Echocardiography 200518 1440-1463. (doi:10.1016/j.echo. 2005.10.005)

21 Ganau A, Devereux RB, Roman MJ, de Simone G, Pickering TG, Saba PS, Vargiu P, Simongini I \& Laragh JH. Patterns of left ventricular hypertrophy and geometric remodeling in essential hypertension. Journal of the American College of Cardiology 199219 1550-1558. (doi:10.1016/0735-1097(92)90617-V)

22 Devereux RB, Alonso DR, Lutas EM, Gottlieb GJ, Campo E, Sachs I \& Reichek N. Echocardiographic assessment of left ventricular hypertrophy: comparison to necropsy findings. American Journal of Cardiology 198657 450-458. (doi:10.1016/0002-9149(86) 90771-X)

23 Quinones MA, Otto CM, Stoddard M, Waggoner A \& Zoghbi WA. Recommendations for quantification of Doppler echocardiography: a report from the Doppler quantification task force of the nomenclature and standards committee of the American Society of Echocardiography. Journal of the American Society of Echocardiography 200215 167-184. (doi:10.1067/mje.2002.120202)

24 Ommen SR, Nishimura RA, Appleton CP, Miller FA, Oh JK, Redfield MM \& Tajik AJ. Clinical utility of Doppler echocardiography and tissue Doppler imaging in the estimation of left ventricular filling pressures: a comparative simultaneous Dopplercatheterization study. Circulation 2000102 1788-1794. (doi:10. 1161/01.CIR.102.15.1788)

25 Connell JM, MacKenzie SM, Freel EM, Fraser R \& Davies E. A lifetime of aldosterone excess: long-term consequences of altered regulation of aldosterone production for cardiovascular function. Endocrine Reviews 200829 133-154. (doi:10.1210/er.20070030)

26 Ainscough JF, Drinkhill MJ, Sedo A, Turner NA, Brooke DA, Balmforth AJ \& Ball SG. Angiotensin II type-1 receptor activation in the adult heart causes blood pressure-independent hypertrophy and cardiac dysfunction. Cardiovascular Research $2009 \mathbf{8 1}$ 592-600. (doi:10.1093/cvr/cvn230)

27 Saruta T, Suzuki H, Handa M, Igarashi Y, Kondo K \& Senba S. Multiple factors contribute to the pathogenesis of hypertension in Cushing's syndrome. Journal of Clinical Endocrinology and Metabolism 198662 275-279. (doi:10.1210/jcem-62-2-275)

28 Sicard RE \& Werner JC. Dexamethasone-induced histopathology of neonatal rat myocardium. In Vivo 19948 353-358. 
29 Brilla CG \& Weber KT. Mineralocorticoid excess, dietary sodium, and myocardial fibrosis. Journal of Laboratory and Clinical Medicine 1992120 893-901.

30 Sheppard KE \& Autelitano DJ. 11 $\beta$-Hydroxysteroid dehydrogenase 1 transforms 11-dehydrocorticosterone into transcriptionally active glucocorticoid in neonatal rat heart. Endocrinology 2002 143 198-202. (doi:10.1210/en.143.1.198)

31 Mihailidou AS, Loan Le TY, Mardini M \& Funder JW. Glucocorticoids activate cardiac mineralocorticoid receptors during experimental myocardial infarction. Hypertension 200954 1306-1312. (doi:10.1161/HYPERTENSIONAHA.109.136242)

32 Di Cori A, Bongiorni MG, Zucchelli G, Soldati E, Falorni M, Segreti L, Gemignani C, Siciliano A, Bovenzi FM \& Di Bello V. Early left ventricular structural myocardial alterations and their relationship with functional and electrical properties of the heart in myotonic dystrophy type 1. Journal of the American Society of Echocardiography 200922 1173-1179. (doi:10.1016/j.echo. 2009.07.004)

33 Shan K, Bick RJ, Poindexter BJ, Shimoni S, Letsou GV, Reardon MJ, Howell JF, Zoghbi WA \& Nagueh SF. Relation of tissue Doppler derived myocardial velocities to myocardial structure and betaadrenergic receptor density in humans. Journal of the American College of Cardiology 200036 891-896. (doi:10.1016/S07351097(00)00786-5)

34 Ciulla MM, Paliotti R, Esposito A, Cuspidi C, Muiesan ML, Rosei EA, Magrini F \& Zanchetti A. Effects of antihypertensive treatment on ultrasound measures of myocardial fibrosis in hypertensive patients with left ventricular hypertrophy: results of a randomized trial comparing the angiotensin receptor antagonist, candesartan and the angiotensin-converting enzyme inhibitor, enalapril. Journal of Hypertension 200927 626-632. (doi:10.1097/HJH.0b013e3283232838)

35 Ciulla MM, Paliotti R, Esposito A, Dìez J, López B, Dahlöf B, Nicholls MG, Smith RD, Gilles L, Magrini F \& Zanchetti A. Different effects of antihypertensive therapies based on losartan or atenolol on ultrasound and biochemical markers of myocardial fibrosis: results of a randomized trial. Circulation 2004110 552-557. (doi:10.1161/01.CIR.0000137118.47943.5C)

36 Diez J, Laviades C, Mayor G, Gil MJ \& Monreal I. Increased serum concentrations of procollagen peptides in essential hypertension: relation to cardiac alterations. Circulation 199591 1450-1456. (doi:10.1161/01.CIR.91.5.1450)

37 Yamamoto K, Mano T, Yoshida J, Sakata Y, Nishikawa N, Nishio M, Ohtani T, Hori M, Miwa T \& Masuyama T. ACE inhibitor and angiotensin II type 1 receptor blocker differently regulate ventricular fibrosis in hypertensive diastolic heart failure. Journal of Hypertension 200523 393-400. (doi:10.1097/00004872200502000-00022)

Received 8 July 2011

Revised version received 19 September 2011

Accepted 17 October 2011 